\title{
Formación de profesionales en entretenimiento
}

\section{Training of entertainment professionals}

Carlos Cienfuegos Alvarado*

Universidad Anáhuac México

Av. Universidad Anáhuac núm. 46, Col. Lomas Anáhuac, Huixquilucan, Estado de México, C.P. 052786, México

Giselle Escalante Castillo**

Universidad Anáhuac México

Av. Universidad Anáhuac núm. 46, Col. Lomas Anáhuac, Huixquilucan, Estado de México, C.P. 052786, México

Editor: Rogelio del Prado Flores

Fecha de recepción: 21 de julio de 2021

Fecha de aceptación: 25 de agosto de 2021 carlos.cienfuegos@anahuac.mx https://orcid.org/0000-0001-9228-1484 giselle.escalante@anahuac.mx
https://orcid.org/0000-0001-8128-6212

https://doi.org/10.36105/stx.2021edesp50aniv.09

\section{RESUMEN}

Se analiza el origen, las razones y circunstancias que motivaron la creación del programa de la Licenciatura en Dirección de Empresas de Entretenimiento, dentro de la Facultad de Comunicación, en el marco de su 50 aniversario. Con una mirada hacia el pasado y presente, revisa las aportaciones que el programa ha hecho a la industria, a través de la formación de profesionales en entretenimiento. Se describe el entretenimiento en el siglo XXI y enfatiza las características del egresado de la licenciatura para, finalmente, ofrecer una radiografía tanto del programa como de la infraestructura tecnológica que ha hecho posible que esta licenciatura sea hoy -a casi I 2 años de su apertura- uno de los programas más reconocidos en nuestro país por su calidad académica.

Palabras clave: formación, entretenimiento, industria, profesionales, tecnología.

\footnotetext{
* Director de la Facultad de Comunicación de la Universidad Anáhuac México. Cuenta con una maestría en Dirección de Empresas del Instituto Panamericano de Alta Dirección de Empresa (IPADE) y es candidato a Doctor en Administración Pública por la Universidad Anáhuac México.

** Coordinadora de la Licenciatura en Dirección de Empresas de Entretenimiento, cuenta con una maestría en Comunicaciones Corporativas con especialidad en Branding y es candidata a Doctor en Comunicación Aplicada por la Universidad Anáhuac México.
} 
ABSTRACT

Analyzes the origin, reasons and circumstances that motivated the creation of the Bachelor of Entertainment Business Management program, within the Faculty of Communication, within the framework of its 50 th anniversary. With a look to the past and present, he reviews the contributions that the program has made to the industry, through the training of entertainment professionals. It begins with a brief description of entertainment in the 2Ist century and emphasizes the characteristics of the bachelor's degree graduate to, finally, offer an x-ray of both the program and the technological infrastructure that has made it possible for this bachelor's degree to be today -almost I2 years old. of its opening- one of the most recognized programs in our country for its academic quality.

Keywords: training, entertainment, industry, professionals, technology.

\section{ESPACIOS DE TIEMPO LIBRE, ESPARCIMIENTO Y RECREACIÓN}

En el siglo XXI, hablar de entretenimiento es hablar de una industria en constante crecimiento, de aquellas experiencias individuales y colectivas que se dan en los espacios de tiempo libre, esparcimiento y recreación, donde las personas buscan salir de una realidad que les ha sido impuesta por las sociedades posmodernas.

Desde la aparición del ser humano, el entretenimiento ha sido parte esencial de su vida y desarrollo. A lo largo de la historia, el entretenimiento ha transitado por diversas etapas de evolución, que van desde los medios análogos a los servicios en streaming, de los conciertos masivos a los festivales de música, de los parques temáticos a la realidad virtual, de la museografía conservadora al arte inmersivo, de las videoconsolas de primera generación a los videojuegos free to play.

Hoy en día, no existe un solo ser humano que escape a la oferta de entretenimiento global y su vínculo con las tendencias y los rasgos más visibles de la vida social contemporánea; es decir, a aquello que somos, lo que hacemos, donde vamos, de lo que hablamos y nos interesa.

El desarrollo de contenidos en las diversas formas de entretenimiento afecta las emociones, actitudes y conductas de las audiencias, que a través de experiencias multisensoriales permiten al público oscilar entre la euforia y la emoción, la cultura y el arte, la imaginación y el aprendizaje.

Las artes escénicas, los eventos masivos, la industria editorial, los videojuegos y la música, entretienen, culturizan, divierten y saturan el tiempo libre, pero también tocan sentimientos, infunden valores, despiertan pasiones, generan tendencias, democratizan conteni- 
dos y muestran modelos de comportamiento que, en ocasiones, se convierten en símbolos generacionales.

La Licenciatura en Dirección de Empresas de Entretenimiento, creada hace nueve años, se ha consolidado como pionera en la formación del estratega especializado en el ámbito del entretenimiento, una industria en constante crecimiento alrededor del mundo. Gracias a su programa novedoso y lleno de posibilidades, ha tenido gran aceptación, tanto en estudiantes como en el mercado de trabajo, que hoy requiere de profesionistas cada vez mejor preparados.

\section{FORMACIÓN DE LÍDERES DE ENTRETENIMIENTO}

En un país como México, con una enorme diversidad y riqueza cultural, donde los avances tecnológicos y el uso de redes, sumados al dinamismo de las nuevas generaciones para la difusión de esa cultura y para la incorporación de audiencias de diversos tipos a través de nuevas formas de producción artística, abre nuevos horizontes a los egresados de la licenciatura.

El espíritu emprendedor que el programa y la misma universidad generan, expande la capacidad de ofrecer formas creativas y originales de organización, producción y ampliación de públicos en un país que, con una gran tradición artística, por un lado ofrece numerosas oportunidades de negocios en la industria del espectáculo y, por el otro, requiere acercar a grandes sectores de la población a las distintas manifestaciones del arte popular y de la cultura en general.

Tomando en cuenta este contexto, la Licenciatura en Dirección de Empresas de Entretenimiento ha creado un programa novedoso, audaz y lleno de posibilidades, que ha asombrado por su rápido crecimiento y por la aceptación que ha encontrado tanto en estudiantes con creatividad e iniciativa, como en un mercado de trabajo en expansión, que hoy requiere de profesionistas especializados y cada vez mejor preparados para dar respuesta a necesidades muy diversas de recreación y esparcimiento.

En este sentido, las trece generaciones que hasta mayo de 202I han egresado, cuentan con una formación sólida e integral, que les proporciona un conocimiento amplio tanto en la esfera económico-administrativa como en la histórico-social; además de desarrollar competencias analíticas y de toma de decisiones, siempre alineadas a los valores que dan fundamento a la formación Anáhuac, como son el liderazgo de acción positiva y el humanismo cristiano.

La formación basada en valores ha sido un pilar de nuestra licenciatura, por lo que se han generado espacios para asegurar el desarrollo intelectual, humano, social y espiritual 
que busca, ante todo, la innovación en tendencias artísticas, culturales, deportivas y comerciales, así como de los gustos de las audiencias. Está plenamente identificado con las nuevas tecnologías, detecta y promueve talento, organiza eventos y produce contenidos, productos y servicios de vanguardia procurando un sano entretenimiento. Su mayor especialización es la gestión, administración e innovación de negocios dentro de la industria, siempre visualizando el mayor beneficio para la sociedad.

Asimismo, se ha buscado perfilar al Director de Empresas de Entretenimiento Anáhuac como un profesionista generador de experiencias, que le permitan a las audiencias mejorar su calidad de vida, a través de contenidos creativos y originales, que buscan siempre la democratización de la cultura.

De la experiencia de las primeras generaciones, de la percepción de los profesores y del propio mercado de trabajo, se desprenden importantes posibilidades que abren nuevas áreas de oportunidad y que satisfacen necesidades vitales para el mercado actual, dando respuesta a puestos de trabajo que antes no estaban profesionalizados.

Con el programa de la licenciatura, se busca que los distintos ámbitos de la industria del entretenimiento y ocio puedan aspirar a un fin mayor, que vaya más allá de la diversión y la ganancia económica; que los productos que se generen dentro de la industria estén orientados a ayudar a la sociedad en cada una de sus facetas:

a) En lo económico. Porque en la medida en que las producciones fomenten una competencia sana y responsable, que derive en una mayor y mejor calidad en las empresas de entretenimiento, promoviendo en las empresas la responsabilidad social, se podrá contribuir en la justicia social y la retribución a la sociedad.

b) En lo cultural. Porque en la medida en que la industria del entretenimiento sea capaz -a través del arte, la música, el teatro y la literatura- de cumplir con la meta de democratizar la cultura, promoviendo la participación de la sociedad en este tipo de actividades, será posible aspirar al desarrollo humano a través del conocimiento, la sabiduría y la belleza. Los medios de entretenimiento, si apuestan por la ética, podrán llevarnos a entender las prácticas culturales y las tradiciones de los demás pueblos; así como a valorar nuestro patrimonio cultural y preservarlo.

c) En lo educativo. Porque la diversidad de producciones y eventos que se generan en la industria del entretenimiento tienen la capacidad de ofrecer herramientas e instrumentos complementarios a la formación de niños, adolescentes, padres de familia y ancianos. Gracias a su nivel de penetración, el eduentretenimiento puede influir en los sectores más marginados y ofrecerles una visión esperanzadora del mundo. Si se emplean adecuadamente, podrían ser fuente de progreso social. 
d) En lo personal. Porque la sociedad tiene la necesidad de contar con espacios de esparcimiento, en los que pueda enriquecer la experiencia vital al transmitir mensajes, noticias, ideas y acontecimientos positivos que inspiren, alienten y lleven a las personas a participar en hechos trascendentales. Tanto la formación intelectual, como cultural y espiritual, se puede beneficiar de palabras e imágenes constructivas.

\section{LA VINCULACIÓN, CLAVE DE LA EMPLEABILIDAD}

Sin lugar a dudas, uno de los principales objetivos dentro de la licenciatura ha sido ofrecer actividades académicas, espacios de diálogo entre los principales actores y directores de las empresas de entretenimiento más importantes y reconocidas, tanto a nivel nacional como internacional; lo anterior en el afán de ofrecerles una visión mucho más cercana a la realidad del mercado de trabajo, a través del conocimiento, experiencia, historia y buenas prácticas por parte de los gigantes del entretenimiento en México.

Siguiendo esta misma línea de vinculación y empleabilidad, semestre a semestre, la licenciatura convoca a grandes personalidades dentro del negocio del entretenimiento como son:

- 20II: Consuelo Salazar, presidenta del Conaculta.

- 20I2: Pedro Torres, productor estelar de realitys de Televisa.

- 20I3: Lic. Robert Stephen Lear Clisby, exdirector de EMI Music.

- 20I4: Mtro. José Ramón Fernández Álvarez, comentarista deportivo; Sr. Arturo Velasco, director de Televisa Música.

- 2015: Dr. Gerardo Estrada Rodríguez, coordinador ejecutivo del Auditorio Nacional y Lic. Francisco Serrano Carreto, director de Desarrollo Corporativo del Auditorio Nacional; Mtro. Sergio Félix Ramírez Cárdenas, subdirector del Instituto Nacional de Bellas Artes.

- 20I6: Lic. Ricardo Pérez Teuffer Fournier, vicepresidente de Comercialización de Grupo Televisa; Lic. Luis de Llano Macedo, productor ejecutivo de Televisa.

- 20I7: Mtro. Ricardo Cayuela Gally, director editorial de Penguin Random House Mondadori.

- 20I8: Tom Wages, Co-Executive Director de la International Association of Amusement Parks and Attractions Foundation.

- 20I9: Mtro. Xavier López Ancona, presidente y fundador de Kidzania. 
Cabe destacar que estos espacios de diálogo entre los protagonistas de la industria del entretenimiento y los alumnos son una muestra de la capacidad que tiene la licenciatura de desarrollar lazos permanentes con el universo de empleadores potenciales, para así realimentar los desafíos técnicos y profesionales en la industria del entretenimiento. Ejemplo de lo anterior son los convenios de colaboración y prácticas que hasta la fecha suman más de 400; cada uno de ellos se ha establecido con diversas empresas, instituciones y organizaciones líderes dentro de la industria, en el afán de permitir que nuestros alumnos puedan ampliar sus horizontes fuera de la universidad. De esta estrategia académica se han generado una serie de voluntariados, tanto a nivel nacional como internacional, de tal manera que los alumnos de la licenciatura hoy tienen acceso a experiencias laborales dentro de algunas empresas líderes en la industria como son:

- OCESA: Corona Capital, EDC, Vive Latino y Fórmula I.

- Auditoria Nacional: Las Lunas del Auditorio.

- Distrito Global: Cumbre Tajín.

- Jardines de México.

- Arena Ciudad de México

- The Global Association for the Attractions Industry (IAAPA).

Como es natural, en un mundo globalizado y competitivo, surgen dos términos que se han convertido en factores de producción: globalización e internacionalización, ambos utilizados para describir las tendencias hacia la intensificación en las relaciones globales de interacción e intercambio, la interconexión mundial en los campos de la comunicación social y la armonización transnacional de los modelos y las estructuras sociales. En síntesis, ambos términos se refieren a una realidad social que se extiende cada vez más a experiencias individuales.

En este sentido y como respuesta a la tendencia mundial, el programa ofrece opciones diversas de internacionalización, a través de la movilidad, de tal suerte que cada semestre el número de alumnos que viajan a universidades alrededor del mundo se incrementa.

La Universidad Católica de Lyon (Francia), el London College of Fashion (Inglaterra), la Universidad del Sagrado Corazón en Milán (Italia), el Royal Melbourne Institute of Technology (Australia), la Universidad Complutense de Madrid (España) y el Disney International College Program en Central Michigan University (Estados Unidos), son solo algunos ejemplos de la manera en que la Licenciatura en Dirección de Empresas de Entretenimiento promueve la movilidad estudiantil internacional, dado que reconoce en ésta prác- 
tica a uno de los componentes de la educación media superior que se enmarca en el urgente requerimiento que enfrentan las instituciones, al fomentar la cooperación internacional e impulsar su internacionalización como una prioridad estratégica de cara al siglo XXI.

Entre muchos otros beneficios, la internacionalización a través de la movilidad estudiantil les permite extender sus horizontes académicos y personales; desarrollar una capacidad de adaptación a situaciones nuevas y complejas, haciéndolos más tolerantes a la diferencia; los hace más competitivos, dado que les permite acceder a nuevas tecnologías y diferentes sistemas educativos, conociendo distintas metodologías de enseñanza y estudio. Finalmente, este tipo de prácticas le permite a la licenciatura crear vínculos académicos para la creación de proyectos conjuntos de colaboración en todos los niveles: estudiantil y docente.

\section{ESTADÍSTICAS DE INGRESO Y EGRESO}

En la actualidad, la Licenciatura en Dirección de Empresas de Entretenimiento cuenta con 500 alumnos inscritos en el programa. Por otra parte, con la finalidad de ofrecer programas de educación continua dentro del Campus Anáhuac México, en el 2015, se abrió el Diplomado en Wedding Planner, y en el 2018 el de Gestión en Imagen y Styling, dentro de los cuales se han formado a 80 y 40 alumnos, respectivamente.

A la fecha, la Licenciatura en Dirección de Empresas de Entretenimiento (LDEE) ha transitado por tres planes de estudio: el original que data del 20Io, el plan 2016 y el tercer plan de estudios 2020. Cabe destacar que, al día de hoy, tan solo en Campus Norte han ingresado 825 alumnos. Su buena aceptación y resultados, en términos de crecimiento de matrícula y trayectorias escolares a lo largo de once generaciones de alumnos (2010-20I9), ha motivado la apertura de la carrera a la oferta académica de los campus: México Sur, Puebla, Querétaro, Jalapa y Cancún de la propia Universidad Anáhuac.

Además cuenta con un cuerpo académico de más de 40 profesores, la mayoría de los cuales tienen un grado de maestría y doctorado, todos ellos con formación y experiencia en el ámbito del entretenimiento; en este sentido, se debe reconocer el esfuerzo que la coordinación de la licenciatura realiza para lograr el reclutamiento de profesores que se han formado en la práctica, por lo que tienen la ventaja de seguir desarrollándose en el campo profesional, lo cual incluso les permite vincular activamente a los alumnos y egresados del programa con sus campos de trabajo. 
INFRAESTRUCTURA TECNOLÓGICA

Al respecto, es importante destacar que los laboratorios, estudios y equipo con los que cuenta la licenciatura son de los mejores en América Latina: el Estudio de Grabación Armando Ávila, que cuenta con un mezclador de 24 canales y un sistema 5.I, con el cual los alumnos comprenden los procesos de creación y producción de un proyecto musical; el Laboratorio de Motion Capture con tecnología de vanguardia y un equipo de producción de eventos de música en vivo.

Esta infraestructura tecnológica ha permitido que los alumnos del programa puedan contar con una formación tanto teórica como práctica, satisfaciendo necesidades importantes para el mercado actual, y dando respuesta a puestos de trabajo que antes no estaban profesionalizados.

Con la finalidad de mantener el programa a la vanguardia tecnológica, para el año 2025 se tiene proyectada la construcción del Laboratorio de Análisis de Movimiento con Inteligencia Artificial, con el que se comenzará a formar de manera multidisciplinar, tanto a los alumnos de la Facultad de Comunicación, como a los de otras facultades como Medicina, Ingeniería, Diseño y Arquitectura.

\section{ACREDITACIONES}

Las acreditaciones son un proceso de revisión de los programas académicos y de su operación, con el fin de mejorar la calidad de las instituciones, de sus planes y programas. En este sentido, las acreditaciones buscan fomentar entre las instituciones de educación superior una cultura de mejora continua, propiciando que los programas académicos alcancen parámetros de calidad, tanto nacionales como internacionales.

En el año 2017, la Licenciatura en Dirección de Empresas de Entretenimiento obtuvo la acreditación por parte de la Asociación para la Acreditación y Certificación en Ciencias Sociales (ACCECISO), órgano reconocido por el Consejo para la Acreditación de la Educación Superior (COPAES), cuya función es llevar a cabo procesos de evaluación y acreditación de los programas y planes de estudios en las disciplinas de las Ciencias Sociales.

La acreditación al programa académico de la Licenciatura en Dirección de Empresas de Entretenimiento, lo reconoce como pionero en la creación de un espacio de formación de profesionales en la dirección de proyectos culturales, los cuales se orientan en una lógica de la pertinencia social, a partir de la práctica comunicativa; al mismo tiempo, destaca la naturaleza innovadora del programa, así como su capacidad de crecimiento en el contexto 
nacional, lo que permite la emergencia de una amplia gama de posibilidades de desarrollo para los estudiantes, al convertirlos en profesionistas capaces de dar respuesta a las demandas de recreación y entretenimiento.

Al obtener la acreditación de ACCECISO, se reconoció a la Licenciatura en Dirección de Empresas de Entretenimiento como un programa que cumple con criterios, indicadores y parámetros de calidad, tanto en su estructura, organización, funcionamiento e insumos, como en sus procesos de enseñanza y servicios, y que por lo mismo tiene pertinencia social.

\section{EVOLUCIÓN}

La industria del entretenimiento engloba distintos factores dentro de los mercados más importantes a nivel nacional, ha contribuido en el crecimiento económico, creación de nuevos empleos, así como al desarrollo dinámico de la sociedad. Contribuye con $8.0 \%$ del Producto Interno Bruto (PIB), registra tasas de crecimiento de $\mathrm{I} 8 \%$ anual y el pago a los trabajadores del sector es superior en $30 \%$, en promedio, en comparación con otros sectores, de acuerdo con el director general de Pixelatl Festival, animación, videojuegos y cómic, José Iñesta Ocampo.

El Global Entertainment and Media Outlook 2016-2020 revela que la industria goza de un crecimiento estable. De hecho, en 36 de los 54 países cubiertos por el estudio, el gasto de la Industria de Entretenimiento y Medios está aumentando más rápidamente que el PIB, generalmente por un factor superior al 50\% (PWC, 2017).

Tomando en cuenta estos datos y la visión a futuro dentro de la industria, el año 2016 marcó una nueva etapa en la vida de la Licenciatura en Dirección de Empresas de Entretenimiento. El reto era grande: alumnos, padres de familia, empresas dentro de la industria y sociedad en general demandaban la formación de recursos humanos altamente competitivos y profesionales, capaces de aportar valor tanto a la industria del entretenimiento en nuestro país, como a la sociedad que consume los productos.

Por tal razón, se realizó un cambio importante en el programa de estudios, al crear seis áreas profesionales, en el afán de proporcionar al egresado las competencias y habilidades necesarias para poder integrarse a un mundo laboral cada vez más competitivo. En este sentido, se introdujeron tres cursos de corte sociohistoriográfico con la intención de enriquecer la formación de nuestros alumnos:

- Introducción al negocio del entretenimiento

- Origen de la industria del entretenimiento en México

- Desarrollo de la industria del entretenimiento en México 
Se fortalecieron los temarios con una actualización en los abordajes intangibles, buscando sinergia con Economía y Negocios y Comunicación, desde el punto de vista digital y manejo de software. Asimismo, se crearon vinculaciones para que además de contar con la especialización se tuvieran de manera extracurricular actividades en las que los alumnos estuvieran en escenarios reales de trabajo como voluntarios en diferentes espacios como: Las Lunas del Auditorio, Premios Miau, Premios Ariel, Cumbre Tajín, Corona Capital, Vive Latino EDC y Pal Norte, entre otros.

De la misma manera y en el afán de fortalecer la internacionalización de nuestros alumnos y egresados, se lograron convenios con The Global Association for the Attractions Industry (IAPPA), de tal suerte que los alumnos de la Licenciatura en Dirección de Empresas de Entretenimiento han podido participar en su convención anual celebrada en la ciudad de Orlando, Florida, para realizar voluntariados que les otorgan no solo experiencia laboral, sino también un amplio conocimiento de la industria a nivel mundial, al poder accesar a sus cursos y conferencias.

\section{CONCLUSIONES}

En el siglo XXI, los avances tecnológicos y la gran variedad de contenidos han llevado a las sociedades posmodernas a nuevas formas de consumir entretenimiento. La era digital ba revolucionado esta industria, que abora está en el centro de la vida de las personas. En este contexto, nuestro rol boy es ofrecer un programa de estudio capaz de generar egresados que puedan insertarse en un mercado laboral con un gran impacto social y económico.

Los logros alcanzados, el prestigio y reconocimiento adquirido, la imagen de madurezy calidad proyectada dentro de la industria del entretenimiento en México, el profesionalismo y preparación de los alumnos, aunados a la pasión por la creación de productos y servicios que eleven la calidad de vida de la sociedad mexicana, ofrecen un futuro prometedor para la Licenciatura en Dirección de Empresas de Entretenimiento. Un futuro que implica responder día a día a las expectativas de un mercado cada vez más competitivo y en constante crecimiento, a fin de seguir contribuyendo a la construcción de un México mejor, un país donde se promueva y respete la democratización de la cultura.

En agosto de 2020, se cumplieron Io años de haber lanzado la Licenciatura en Dirección de Empresas de Entretenimiento, para lo cual se tuvo la segunda actualización del plan de estudios, el cual tiene una combinación de las seis áreas profesionales dentro de su bloque profesional obligatorio, y la inclusión de cursos orientados a la instrucción en diseño, creación y análisis del uso de tecnología de comunicación inmersiva, marketing experiencial, am- 
bientes virtuales, experiencias ${ }_{3} \mathrm{D}$ e inteligencia artificial. Así como la oferta de tres minors para las demás licenciaturas de la Universidad Anáhuac México, con una temática enfocada en la producción de eventos, comunicación digital e inteligencia artificial.

Con lo anterior, se mantiene la estrategia de estar en un continuo estudio y actualización de la práctica enseñanza-aprendizaje sobre las industrias creativas, que aseguren una formación actualizada de los futuros líderes especializados en empresas de arte, cultura y entretenimiento.

\section{REFERENCIAS}

Caniem. (2015). Comercialización de libros impresos. http://www.caniem.com/es/content/camara-nacional-de-la-industria-editorial-mexicana

El Sol de México. (2019, 20 de abril). Streaming ya abarca el $47 \%$ de la industria musical. https://www.elsoldehermosillo.com.mx/finanzas/tecnologia/streaming-ya-abarca-el-47-dela-industria-musical-3344902.html

IFPI. (2019). IFPI Global Music Report 2019. https://www.ifpi.org/news/IFPI-GLOBAL-MUSIC-REPORT-2019

Monitor Latino. (2017). Top Managers 2017. http://monitorlatino.com/top-managers-20I6/

Universidad Anáhuac. (2016). Somos Anáhuac México. Líderes de Acción Positiva. Universidad Anáhuac México. https://www.anahuac.mx/mexico/mision

Pollstar. (2018). Year end, Worldwide ticket sales top Ioo promoters. https://www.pollstar.com/ Chart/20I8/12/20I8YearEndWorldwideTicketSalesTopıooPromoters_69ı.pdf

ProMéxico. (2018). La industria de los videojuegos en México. http://mim.promexico.gob.mx/ work/models/mim/templates-new/Publicaciones/Articulos/La-industria-videojuegos-Mexico.pdf

PWC. (2017). Entertainment and Media Outlook México 2016-2020. Publicaciones Entertainment and Media Outlook México. https://www.pwc.com/mx/es/publicaciones/c2g/entertainment-and-media-outlook-mexico-20I6-2020.pdf

Esta obra está bajo Licencia Creative Commons Atribución-NoComercial-SinDerivadas 4.o Internacional. 